\title{
Cluster algorithm for non-additive hard-core mixtures
}

\author{
Arnaud Buhot \\ UMR 5819 (UJF, CNRS, CEA) DRFMC/SI3M, CEA Grenoble, \\ 17 rue des Martyrs, 38054 Grenoble cedex 9, France
}

\begin{abstract}
In this paper, we present a cluster algorithm for the numerical simulations of non-additive hardcore mixtures. This algorithm allows one to simulate and equilibrate systems with a number of particles two orders of magnitude larger than previous simulations. The phase separation for symmetric binary mixtures is studied for different non-additvities as well as for the Widom-Rowlinson model (B. Widom and J. S. Rowlinson, J. Chem. Phys. 52, 1670 (1970)) in two and three dimensions. The critical densities are determined from finite size scaling. The critical exponents for all the non-additivities are consistent with the Ising universality class.

PACS numbers: 61.20.Ja, 64.60.Hr, 05.10.Ln
\end{abstract}

\section{INTRODUCTION}

The Widom-Rowlinson (WR) model [1] attracted a lot of attention as a prototype for the liquid-vapor phase separation. This simple model is composed of a twocomponent system where likewise particles do not interact whereas unlike particles interact through a hard-core potential. It was shown to present a phase separation at high density and a critical point that belongs to the Ising universality class [2, 3]. This mixture exhibits a liquidliquid critical point (with large composition fluctuations) in contrast to pure fluids that experience a liquid-vapor critical point (with large density fluctuations). However, the phase transitions are related [4] and believed to depend on the same universality class [5].

A straightforward generalization of the WR model is the non-additive hard-core (NAHC) mixtures where the likewise particles also experience a hard-core interaction. Two particles $i$ and $j$ present a minimal distance of approach $\sigma_{X Y}$ with $X$ and $Y$ representing respectively the $A$ or $B$ component from which belong particles $i$ and $j$. Non-additive mixtures are characterized by $\sigma_{A B}=$ $\left(\sigma_{A A}+\sigma_{B B}\right)(1+\Delta) / 2$ with $\Delta \neq 0$. Additive mixtures correspond to $\Delta=0$. For a negative non-additivity $\Delta<0$, particles tend to form hetero-coordinations [6, 7]. For a positive non-additivity $\Delta>0$, an entropically driven phase separation occurs between two phases at sufficiently high density due to the extra repulsion between unlike particles [8]. The two phases are chemically different, one is rich in $A$ particles whereas the other is rich in $B$ particles. This phase separation occurs even for the symmetric mixtures where $\sigma_{A A}=\sigma_{B B}$. The critical density as well as the universality class have been determined from different numerical simulations [5, 9, 10, 11, 12. The special case of $\sigma_{A A}=\sigma_{B B}=0$ corresponding to the WR model has also been studied 2, 3]. Additive mixtures with a strong asymmetry $\sigma_{A A} \ll \sigma_{B B}$ are also of interest and present another kind of entropically driven phase separation transition predicted for the first time by Biben and Hansen 13 but only recently observed by numerical simulations 14, 15].

Most recent simulations of the NAHC mixtures 5, 11,
12 used a Monte-Carlo algorithm within the semigrand canonical ensemble [16, 17]. In addition to the simple moves of particles as simple Monte-Carlo steps, some steps consist in changing the nature (or component) of a particle when the hard-core interactions permit this modification. The resulting ensemble corresponds to a fixed total number of particles but a variable composition (or fixed difference of chemical potentials between particles of different components). A detailed description of the algorithm in the case of binary mixtures with squared-well interactions may be found in the paper of de Miguel et al. 17]. Working in the semigrand canonical ensemble gives access to the coexistence curve of the model. However, the algorithm is limited to rather small system sizes. The largest simulations concerned 16384 particles 11 but most of them were limited to a few thousands 9, 10, 12. Furthermore, only small nonadditivities $\Delta \leq 1$ have been simulated [11, [12]. The main reason for this limitation is the following. When a Monte-Carlo step corresponding to a change of components is tried, at high non-additivity there is a large probability of overlap with at least one of the neighbors. This results in the rejection of the change of components and a dramatic slowing down of the equilibration of the numerical simulations.

In the present paper we consider a cluster algorithm proposed by Dress and Krauth [18] for hard-core mixtures which proved useful for the detection of the phase separation in additive asymmetric mixtures [14, 19, 20] and for the analysis of two dimensional polydisperse hard-core mixtures 21]. The advantage of this algorithm is to allow one to equilibrate systems with up to $10^{6}$ particles (two order of magnitude larger than previous simulations). Also, the slowing down of the equilibration for large non-additivity is completely avoided with this cluster algorithm. This allows us to analyze the limit of infinite non-additivity $(\Delta \rightarrow \infty)$ where the NAHC mixtures converge to the WR model. Furthermore, the coexistence curve is accessible in contrary to the previous cluster algorithm used for the WR model [3].

The paper is organized as follows: in section II, we present the model of non-additive hard-core mixtures and we describe the cluster algorithm used for the numerical 
simulations. We present the numerical results for the two and three dimensional mixtures in section III. From finite size scaling analysis, we extract the critical densities and the critical exponents. Finally, we conclude by a discussion of the results in section IV.

\section{NON-ADDITIVE HARD-CORE MIXTURES}

\section{A. Description of the model}

We consider non-additive hard-core (NAHC) mixtures in two and three dimensions. The system is made of two components $A$ and $B$ with respectively $N_{A}$ and $N_{B}$ particles. The particles experience hard-core interactions. No overlap is possible if the distance $d$ between the center of the particles $i$ and $j$ is lower than $\sigma_{X Y}$ where $X$ and $Y$ represent the components of particles $i$ and $j$ respectively. For convenience, the particles are placed in two identical boxes of equal volume $V$. Periodic boundary conditions are assumed on each of the boxes. From the following description of the cluster algorithm, the reason for considering two boxes will become obvious. Notice that the consideration of two boxes is of common use for the determination of the coexistence curve in semigrand canonical ensemble simulations.

In the following, we restrict ourselves to symmetric mixtures with $\sigma_{A A}=\sigma_{B B}=\sigma$ however we allow for a general positive non-additivity $\Delta=\sigma_{A B} / \sigma-1$. Due to the hard-core interactions, the temperature plays a trivial role and the phase diagram is determined only by the number density $\rho=\rho_{A}+\rho_{B}=N_{A} / 2 V+N_{B} / 2 V$ and the composition $x_{A}=1-x_{B}=\rho_{A} /\left(\rho_{A}+\rho_{B}\right)$ of the system. In the particular case of the symmetric NAHC mixtures, the critical point $\left(\rho_{c}, x_{c}\right)$ is determined in composition $\left(x_{c}=1 / 2\right)$ due to the symmetry. Thus, in the following, we will consider $N_{A}=N_{B}$. Above the critical density $\rho_{c}$, the system separates in two phases $I$ and $I I$. One phase is rich in $A$ particles and the other is rich in $B$ particles. Furthermore, due to the symmetry, those phases are symmetric in composition $\left(x_{A}^{I}=x_{B}^{I I}\right.$ and $\left.x_{A}^{I I}=x_{B}^{I}\right)$.

The determination of the coexistence curve $x_{A}^{I}$ and $x_{A}^{I I}$ is possible from the use of the two equivalent boxes. The overall composition $x_{A}=N_{A} /\left(N_{A}+N_{B}\right)$ is fixed during the simulations whereas the particular compositions $x_{A}^{I}=N_{A}^{I} /\left(N_{A}^{I}+N_{B}^{I}\right)$ and $x_{A}^{I I}=N_{A}^{I I} /\left(N_{A}^{I I}+N_{B}^{I I}\right)$ of the boxes $I$ and $I I$ are free to fluctuate. $N_{X}^{Y}$ corresponds to the number of particles of component $X=A$ or $B$ inside the box $Y=I$ or $I I$. Notice that the number of particles in each box $N^{I}=N_{A}^{I}+N_{B}^{I}$ and $N^{I I}=N_{A}^{I I}+N_{B}^{I I}$ also fluctuates. The ensemble considered is thus intermediate between the grand canonical ensemble and the semigrand canonical ensemble. We will discuss in the following the consequences of the slight density fluctuations inside each box.

In complement to the number density $\rho$, it is interesting to introduce the scaled packing fraction $\eta=v_{A B} \rho$ with $v_{A B}$ the volume of unlike particles. $v_{A B}=\pi \sigma_{A B}^{2} / 4$ in two dimensions and $\pi \sigma_{A B}^{3} / 6$ in three dimensions. This definition of the packing fraction which takes into account the non-additivity allows us to compare the critical packing fraction of the WR model with those of the NAHC mixtures. Notice that from this definition, the packing fraction may exceed one.

\section{B. Description of the cluster algorithm}

A cluster algorithm has been introduced recently by Dress and Krauth [18] for the simulation of hard-core mixtures. Inspired by the lattice cluster algorithms of Swendsen-Wang 22] and Wolff 23], it allows one to equilibrate large off-lattice systems (up to $10^{6}$ particles) thanks to a non-local move of a large number of particles at each Monte-Carlo step. The general idea of the algorithm is to take advantage of the hard-core interactions between particles to construct a cluster of particles that will be moved at each Monte-Carlo step satisfying the detailed balance while keeping ergodicity.

The Monte-Carlo step is constructed as follows:

i) We select randomly one of the two boxes.

ii) A second box is chosen randomly (it can be the same as the previous one).

iii) An inversion symmetry around a randomly chosen pivot point is performed on all the particles of the second box.

iv) The two boxes with their particles are then superimposed on top of each other resulting in a set of clusters of overlapping particles (in the sense of the hard-core interactions).

v) A particle is randomly selected and the cluster from which it belongs is flipped. Each particle belonging to this cluster is moved from its initial box to the other box in the position corresponding to the superimposed configuration.

For a clear graphical representation of a Monte-Carlo step see Figure 1 in Dress and Krauth paper [18].

Let us first prove that the new configuration obtained after the Monte-Carlo step satisfies all the hard-core interactions between particles. a) For two particles outside the flipped cluster there is no overlap since those particles did not move and there was no overlap before the Monte-Carlo step. b) For two particles inside the cluster, they were flipped keeping there relative positions in such a way that there is still no overlap. c) Finally, by construction of the cluster, there is no overlap between a particle belonging to the cluster and one outside the cluster when the two boxes are superimposed and a fortiori after the Monte-Carlo step. Those arguments justify the fact that the new configuration obtained satisfies all the hard-core interactions between particles in each box.

Now let us justify the detailed balance of the cluster algorithm. Each Monte-Carlo step has its symmetric step in the sense that if we start from the final configuration there is a pivot point that gives back the initial configuration. The pivot points being randomly selected with 
a uniform distribution, there are equality between the probabilities to go from the initial to the final configuration and vice-versa. The detailed balance is thus satisfied noting that due to the hard-core interactions both configurations have the same equilibrium probability. In the case of a general potential of interaction between particles, the cluster algorithm may be generalized with the extent of some rejections of the Monte-Carlo steps [24].

On the point ii) of a Monte-Carlo step, either the same box or the other one may be selected. This leads to intra-box or inter-box Monte-Carlo steps. The reason for this choice is to decrease the equilibration time and as we will see to satisfy the ergodicity of the cluster algorithm. With intra-box Monte-Carlo steps (same box selected twice), it has been shown already [14, 18] that the algorithm satisfies internal ergodicity like the usual Monte-Carlo algorithm. By internal ergodicity, we consider the fact that for the given composition of the box, all possible configurations of the particles are attainable by the cluster algorithm. In fact, if the pivot point is chosen sufficiently close to a particle position and this particle considered as the starting point for the cluster construction, the Monte-Carlo step corresponds to a slight move of this particle without affecting the other particles. This move corresponds to a usual Monte-Carlo move in a general algorithm. This argument justifies the internal ergodicity. However, from those moves, the composition of the box is kept constant. In order to change the composition or the relative number of particles of components $A$ or $B$ in each box, inter-box Monte-Carlo steps are necessary. Those inter-box Monte-Carlo steps are performed to exchange the components of particles. If the pivot point is selected such that two particles from different components and boxes superimposed exactly and if one of those particles is selected as the cluster starting point, the Monte-Carlo step reduces to the exchange of those particles. The Monte-Carlo move then corresponds to the usual change of components considered by the MonteCarlo algorithm in the semigrand canonical ensemble. In summary, both usual Monte-Carlo steps (the move of a particle and the change of components) are possible moves in this cluster algorithm justifying the ergodicity if this ergodicity is assumed for the usual Monte-Carlo algorithm in the semigrand canonical ensemble.

As can be seen from the discussion on the ergodicity, the use of the two boxes is useful for the non-additive hard-core mixtures. It has another strong advantage since it allows one to determine the coexistence curve or the relative composition of the two separated phases above the critical density. Due to the symmetry of the problem, the critical point corresponds to an equal partition in particles $A$ and $B\left(x_{c}=1 / 2\right)$ and above the critical density the coexistence curve is symmetric around $x_{c}=1 / 2$. The use of identical boxes is thus justified since the densities $\rho^{I}$ and $\rho^{I I}$ of the two phases are equal. As previously discussed, the total number of particles in each box is slightly fluctuating with this cluster algorithm in contrary to the simulations in the semigrand

\begin{tabular}{|c|c|c|c|}
\hline Exponent & $\nu$ & $\gamma$ & $\beta$ \\
\hline 2D (exact) & 1 & $7 / 4$ & $1 / 8$ \\
\hline 3D (num.) & 0.627 & 1.239 & 0.326 \\
\hline
\end{tabular}

TABLE I: Critical exponents for the Ising universality class in two and three dimensions.

canonical ensemble. However, those fluctuations of the density are not related to the composition fluctuations inside each box. More importantly, around the phase separation transition, the composition fluctuations diverge whereas the density fluctuations stay insensitive to the transition. As a consequence the slight density fluctuations do not affect the coexistence curve determined from the cluster algorithm.

The last question concerning the cluster algorithm concerns the equilibration time or the number of MonteCarlo steps necessary to equilibrate the system. The Swendson-Wang cluster algorithm was introduced to simulate systems of Ising spins with ferromagnetic interactions between neighbor spins on a lattice 22]. In contrary to the simple Monte-Carlo algorithm, this cluster algorithm does not suffer from a critical slowing down at the phase transition due to the fact that the flipped clusters are then directly related to the spin clusters observed around the phase transition. In the case of the present cluster algorithm, such direct relation of the flipped clusters with the configurations of particles is not demonstrated. However, the number of Monte-Carlo steps necessary for the equilibration of the system does not seem to increase significantly when approaching the phase separation transition. It is also interesting to note that this number is roughly independent of the system size as observed for cluster algorithms on lattices and in contrary to usual Monte-Carlo algorithms where this number usually increase strongly with the system size. Another important point is that there is no critical slowing down for the equilibration when the non-additivity is increased. Thus, this cluster algorithm is well adapted for large nonadditivities $\Delta$ and for the WR model in comparison to the Monte Carlo simulations in the semigrand canonical ensemble. In fact, the critical slowing down is observed for small non-addtivities when the phase separation transition occurs at high densities close to a fluid-solid transition.

\section{RESULTS OF THE NUMERICAL SIMULATIONS}

\section{A. Critical exponents and critical packing fractions}

As all second order phase transitions, the critical point in NAHC mixtures is characterized by different critical exponents. From finite size scaling of equilibrium thermodynamic quantities close to the critical point $\left(\rho_{c}, x_{c}\right)$, it is possible to determine those exponents. For the 
NAHC mixtures and for the WR model, the critical exponents are supposed to belong to the Ising universality class (see numerical values of $\nu, \gamma$ and $\beta$ in Table I). In the following, we define different ways to determine or test the value of the critical exponents. We also describe four different ways to define a finite size critical packing fraction $\eta_{c}(L)$ from numerical simulations.

From finite size scaling, it is possible to extract the (infinite size) critical packing fraction $\eta_{c}$ [12, 25]:

$$
\eta_{c}(L)=\eta_{c}-A / L^{1 / \nu}
$$

The finite size of the system $L$ is defined as $N_{A}=L^{d}$ where $d$ is the dimension of the system. The critical exponent $\nu$ is independent of the definition of the finite size critical packing fraction considered in contrary to the coefficient $A$. Due to the corrections to scaling for small system sizes, it is usually difficult to extract the critical exponent $\nu$. However, a linear behavior of the finite size critical packing fraction with the rescaled system size $1 / L^{1 / \nu}$ is a good test of the universality class of the model considered and allows one to extract the critical packing fraction $\eta_{c}$.

The phase separation transition is characterised by the order parameter $m=2\left(x_{A}-x_{c}\right)$ with $-1 \leq m \leq 1$. Its probability distribution $P(m ; \eta, L)$ for a packing fraction $\eta$ and a system size $L$ changes form around the critical packing fraction $\eta_{c}$. In the thermodynamic limit $(L=$ $\infty)$, the distribution of the order parameter presents delta picks. It has a single pick at $m=0$ below the critical packing fraction $\left(\eta<\eta_{c}\right)$. However, above the critical packing fraction $\left(\eta>\eta_{c}\right)$, the distribution is double picked at values $\pm m_{\max }$ with $m_{\max } \sim\left(\eta-\eta_{c}\right)^{\beta}$ for $\eta \gtrsim \eta_{c}$. For finite size systems, the picks broaden but the change of the distribution remains and a maximum $m_{\max }(\eta, L)>0$ may be defined for each packing fraction $\eta$ and size $L$ above the size dependent critical packing fraction $\eta_{c}^{\max }(L)$. The dependence of the maximum $m_{\max }$ on the packing fraction at a fixed system size is [25]:

$$
m_{\max } \sim\left(\eta-\eta_{c}^{\max }(L)\right)^{\beta}
$$

for $\eta \gtrsim \eta_{c}^{\max }(L)$. Knowing the exponent $\beta$, it is thus possible to extract the finite size critical packing fraction $\eta_{c}^{\max }(L)$ from a linear fit of $m_{\max }^{1 / \beta}$ as function of $\eta$. For high packing fraction $\eta, m_{\max }$ saturates to one. This limits the range of packing fractions for which the linear fit is valid. Due to this limited range of the power law behavior, it is difficult to extract the critical exponent $\beta$. However, a linear dependence obtained for the expected value of the critical exponent $\beta$ is still a strong confirmation of the universality class.

Due to the symmetry of the model, the average of the order parameter is zero. However, the mean absolute value of the order parameter is another possibility to define a finite size order parameter:

$$
\langle|m|\rangle(\eta, L)=\int_{-1}^{1}|m| P(m ; \eta, L) d m .
$$

\begin{tabular}{|c|c|c|c|c|c|}
\hline$\Delta$ & 0.5 & 1.0 & 2.0 & $\infty$ & Ising \\
\hline 2D & $1.749(8)$ & $1.749(8)$ & $1.746(7)$ & $1.742(8)$ & $7 / 4$ \\
\hline 3D & $2.03(8)$ & $2.05(8)$ & $2.05(8)$ & $2.05(8)$ & 1.98 \\
\hline
\end{tabular}

TABLE II: Numerical results for the ratio of critical exponents $\gamma / \nu$ for different non-additivities and for the WR model $(\Delta=\infty)$ in two and three dimensions. Numbers in parenthesis correspond to the error on the last digits. The data in column Ising are the predictions of the Ising universality class.

This definition suffers from an additional drawback compared to $m_{\max }$. The average absolute value of the order parameter does not vanish at the finite size critical packing fraction $\eta_{c}^{a v}(L)$. Thus, the power law behavior [25]:

$$
\langle|m|\rangle \sim\left(\eta-\eta_{c}^{a v}(L)\right)^{\beta}
$$

presents corrections to scaling not only for large packing fraction but also around $\eta_{c}^{a v}(L)$. This finite size critical packing fraction $\eta_{c}^{a v}(L)$ may still be extracted from a linear fit of $\langle|m|\rangle^{1 / \beta}$ on a limited range of packing fractions. The small value of the critical exponent $\beta$ in two dimensions leads to a sufficiently large range, however, in three dimensions, the larger value of $\beta$ renders the range of power law behavior (4) too small to be able to extract $\eta_{c}^{a v}(L)$.

The maximum of the modified susceptibility $\chi(\eta, L)=$ $\left\langle m^{2}\right\rangle-\langle|m|\rangle^{2}$ is a third possibility to define a finite size critical packing fraction. This modified susceptibility presents a single maximum in contrary to the real susceptibility $\left\langle m^{2}\right\rangle-\langle m\rangle^{2}$. The general form of the modified susceptibility is [25]:

$$
\chi(\eta, L)=L^{\gamma / \nu-d} \tilde{\chi}\left(L^{1 / \nu}\left(\eta-\eta_{c}\right)\right)
$$

with $\tilde{\chi}(x)$ a function with a single maximum $\tilde{\chi}_{\max }$. The packing fraction at the maximum of the modified susceptibility defines the finite size critical packing fraction $\eta_{c}^{\chi}(L)$. The modified susceptibility may also be used to extract the ratio of critical exponents $\gamma / \nu$. Its maximum depends algebraically on the system size with an exponent $\gamma / \nu-d[25]$ :

$$
\chi_{\max }\left(\eta_{c}^{\chi}(L), L\right) \sim L^{\gamma / \nu-d} \tilde{\chi}_{\max } .
$$

The ratio of critical exponents $\gamma / \nu$ is thus simply determined by the slope of a linear fit in a log-log scale. The results for the two and three dimensional systems are presented in Table II in comparison with the Ising universality prediction. Those results are discussed later.

Another possibility to determine the critical packing fraction concerns the Binder parameter [25]:

$$
U(\eta, L)=1-\frac{\left\langle m^{4}\right\rangle}{3\left\langle m^{2}\right\rangle} .
$$

The Binder parameter saturates to $2 / 3$ for large packing fractions and vanishes for small ones. It also presents the 
interesting property to intersect at the critical packing fraction at least for sufficiently large system sizes. The value $U\left(\eta_{c}, L\right)=U^{*}$ at this intersection is expected to be universal. The monotonous behavior of the Binder parameter may be used to define a finite size critical packing fraction $\eta_{c}^{B}(L)$ as the value for which $U\left(\eta_{c}^{B}(L), L\right)=1 / 2$. The choice of the value $1 / 2$ is arbitrary and could be modified as soon as it is sufficiently different from the boundary values 0 and $2 / 3$ and from the intersction value $U^{*}$. The particular choice of $U^{*}$ would render the finite size packing fraction independent of the system sizes. This point will be discussed further for the three dimensional systems.

We defined different critical packing fractions for finite size systems from the maximum of the modified susceptibility $\eta_{c}^{\chi}(L)$, from the Binder parameter $\eta_{c}^{B}(L)$ and from the coexistence curve either from the maximum of the distribution of the order parameter $\eta_{c}^{\max }(L)$ or from the average absolute value of the order parameter $\eta_{c}^{a v}(L)$. The numerical results obtained from the cluster algorithm are analyzed in the following sections.

\section{B. Two dimensional NAHC mixtures}

In the two dimensional model, we considered four different non-additivities $\Delta=0.5,1.0,2.0$ and 4.0 as well as the WR model $(\Delta=\infty)$. The system sizes ranged from $L=N_{A}^{1 / 2}=10$ to 400 . The largest systems contained $N_{A}+N_{B}=320000$ particles. Numerical simulations were divided in five consecutive runs of $10^{5}$ to $10^{6}$ Monte-Carlo steps depending on the system sizes with longest runs for the largest systems. The first run is kept for equilibration of the initial configuration and the last four runs for the data collection and error estimation.

On Fig. 1] we plot equilibrium configurations for two different packing fractions. The configurations correspond to systems with $N_{A}+N_{B}=3200$ particles and a non-additivity $\Delta=2$. The likewise hard-core diameter $\sigma_{A A}$ and $\sigma_{B B}$ are represented respectively by white and black disks whereas the unlike diameter $\sigma_{A B}$ is represented by light and dark grey disks respectively for the $A$ and $B$ particles. No overlaps between unlike particles are present but overlaps of likewise particles are observed on the grey scale. However, no overlap is present between black and white particles justifying that both configurations satisfy all the hard-core interactions. For the top configuration above the phase separation transtion, $\eta=1.03>\eta_{c}(L) \simeq 0.95$, we observe a large difference between the number of $A$ and $B$ particles. The large percolating cluster of $B$ particles (the dark grey particles) is a clear evidence of the phase separation. On the contrary, on the bottom configuration, $\eta=0.72<\eta_{c}(L)$, the $A$ and $B$ particles are roughly in equal number and perfectly mixed. In this systems, the phase separation did not occur, however, due to the stronger unlike particles hard-core interactions, a local clustering of likewise particles is present.
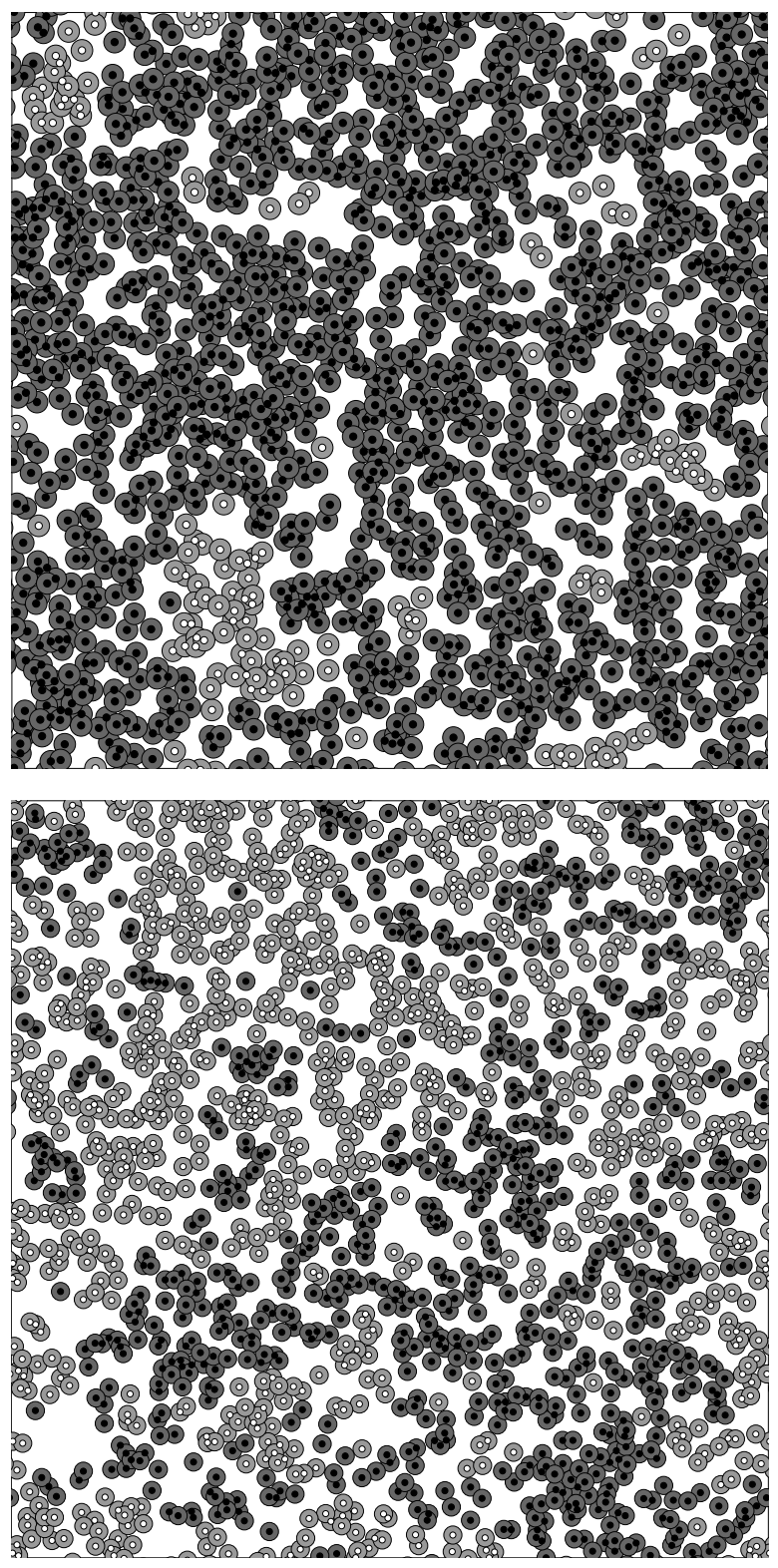

FIG. 1: Equilibrium configurations for a system above (top) and below (bottom) the phase separation transition. The systems correspond to $N_{A}+N_{B}=3200$ particles with a nonadditivity $\Delta=2$. White and black disks correspond to likewise diameter $\sigma_{A A}$ and $\sigma_{B B}$ whereas the light and dark grey disks correspond to the unlike diameter $\sigma_{A B}$. With this representation, the overlaps are only allowed by the hard-core interactions to the likewise particles on the grey scale disks.

The modified susceptibility $\chi$ is determined as function of the packing fraction $\eta$ for different system sizes. It presents a single maximum $\chi_{\max }\left(\eta_{c}^{\chi}(L), L\right)$ at the finite size critical packing fraction $\eta_{c}^{\chi}(L)$. As can be seen on Fig. 2. this maximum depends on the system size with a power law behavior. From a linear fit in a log-log scale, we deduce the ratio of critical exponents $\gamma / \nu$ (see Table III). Due to the corrections to scaling observed for 


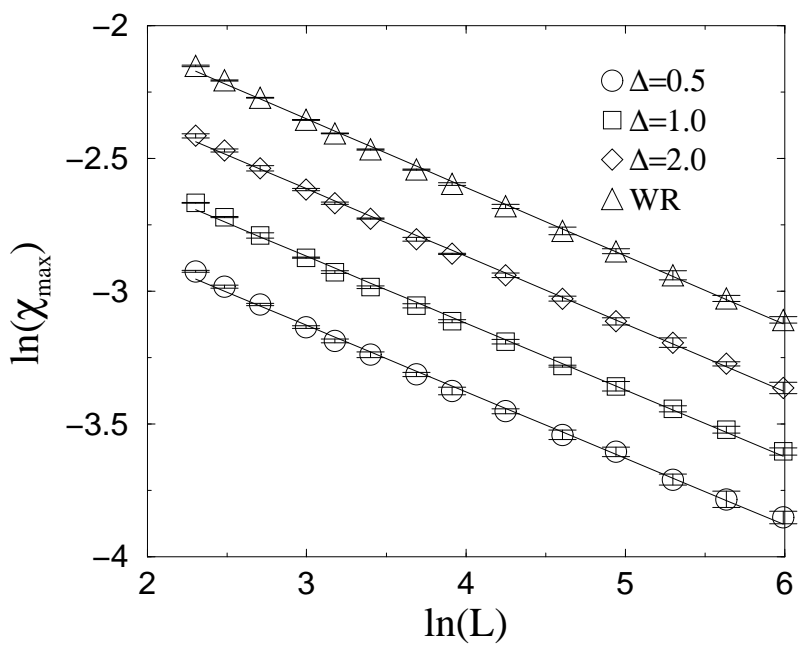

FIG. 2: Maximum of the modified susceptibility $\chi_{\max }$ as function of the system size $L$ in a $\log$ - $\log$ scale for three different non-additivities $\Delta$ and for the WR model (Widom). The ratio of critical exponents $\gamma / \nu$ is determined from linear fits. The three smallest system sizes are removed from the linear fit due to the corrections to scaling effects for those small sizes. The data have been shifted for clarity.

small system sizes, the three smaller sizes $(L=10,12$ and 15) where removed for the linear fit to extract $\gamma / \nu$. This ratio of critical exponents compare nicely with the Ising universality class prediction $7 / 4$ for all the nonadditivities considered and for the WR model. The estimation for the errors presented on Table 1 comprises the error on the maximum of the modified susceptibility and the error coming from the linear fit. The relative error on the ratio of critical exponents $\gamma / \nu$ is smaller than $1 \%$.

The confirmation of the critical exponent $\beta$ is obtained from the two different definitions of the finite size order parameter: $m_{\max }$ and $\langle|m|\rangle$. First, we plot the rescaled maximum of the distribution of the order parameter $m_{\max }^{1 / \beta}$ as function of $\eta$ for different system sizes and for a non-additivity $\Delta=2$ on Fig. 3k. Second, we plot the rescaled average order parameter $\langle|m|\rangle^{1 / \beta}$ for the same system sizes but for a non-additivity $\Delta=1$ on Fig. 3 3 . The linear behavior observed on both figures for a critical exponent $\beta=1 / 8$ confirms the Ising universality class prediction. Similar results are obtained for all the non-additivities and for the WR model. The range of the linear regime is still rather small and limited for large packing fractions to $m_{\max }^{1 / \beta}<0.7$ and $\langle|m|\rangle^{1 / \beta}<0.6$. Furthermore, due to the strictly positive average of the absolute order parameter, the power law behavior is also limited from below to $\langle|m|\rangle^{1 / \beta}>0.1$. The restriction on the range of the power law behavior prevents from a direct determination of the critical exponent $\beta$.

The critical packing fraction $\eta_{c}$ is then determined from the plot of the finite size critical packing fraction $\eta_{c}(L)$ as function of the rescaled system size $1 / L^{1 / \nu}$ with the expected exponent $\nu=1$. On Fig. [4 the numer-
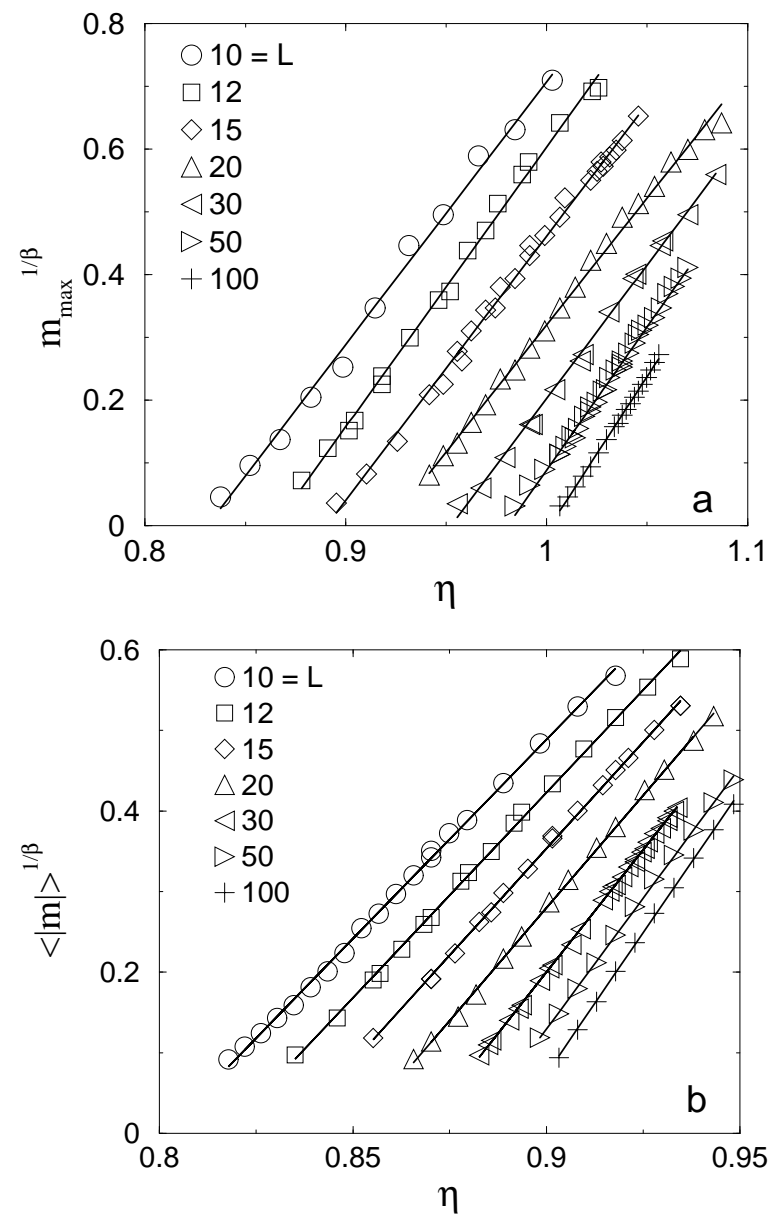

FIG. 3: (a) Rescaled maximum of the distribution of the order parameter $m_{\max }^{1 / \beta}$ for a non-additivity $\Delta=2$ and (b) rescaled average order parameter $\langle|m|\rangle^{1 / \beta}$ for a non-additivity $\Delta=1$ as function of the packing fraction $\eta$ for different system sizes. Linear fits confirm the critical exponent $\beta=1 / 8$ predicted by the Ising universality class and allow us to extract the finite size critical packing fractions $\eta_{c}^{\max }(L)$ and $\eta_{c}^{a v}(L)$ respectively.

ical results for $\eta_{c}^{\max }, \eta_{c}^{a v}$ and $\eta_{c}^{B}$ are plotted for a nonadditivity $\Delta=1$. The results for $\eta_{c}^{\chi}$ close to those for $\eta_{c}^{B}$ have been removed for clarity. The error bars are smaller than the symbols and thus not represented on the figure. As can be seen on Fig. 目 $\eta_{c}^{\max }$ and $\eta_{c}^{a v}$ present a linear behavior with respect to $1 / L^{1 / \nu}$ for $\nu=1$. This confirms the value of the critical exponent $\nu$ predicted by the Ising universality class. However, $\eta_{c}^{B}(L)$ presents corrections to scaling and a quadratic fit is necessary to extract the critical packing fraction $\eta_{c}^{B}$. The same is true for $\eta_{c}^{\chi}$ and similar results are obtained for all the non-additivities as well as the WR model.

All the critical packing fractions from the four different definitions of their finite size analog are presented on Table III for all the non-additivities considered and for the WR model. It is interesting to notice that the four different definitions are not identical since the numerical val- 


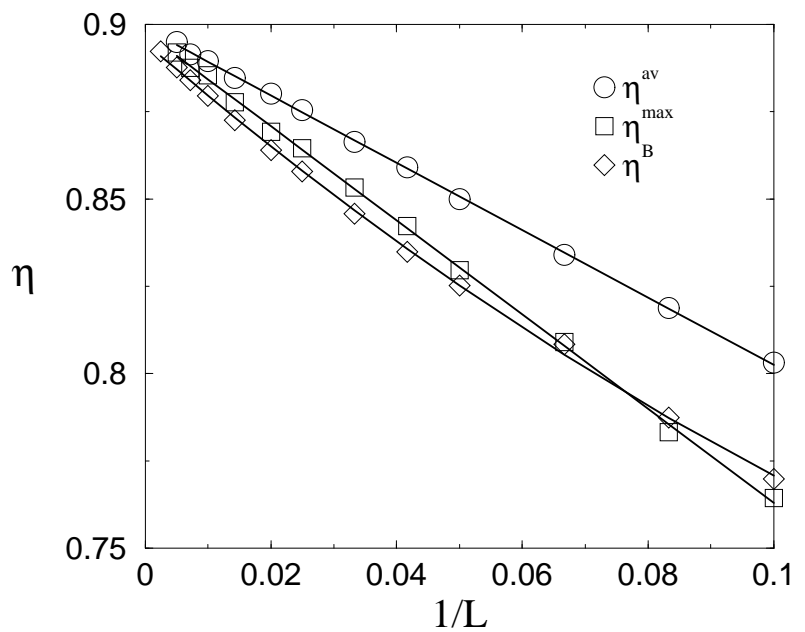

FIG. 4: Finite size critical packing fractions $\eta_{c}^{a v}(L), \eta_{c}^{\max }(L)$ and $\eta_{c}^{B}(L)$ as function of the rescaled system size $1 / L^{1 / \nu}$. The non-additivity considered is $\Delta=1$. Linear fits of $\eta_{c}^{a v}$ and $\eta_{c}^{\max }$ confirm the critical exponent $\nu=1$ of the Ising universality class and allow us to extract the critical packing fractions $\eta_{c}^{a v}$ and $\eta_{c}^{\max }$. A quadratic fit is necessary for $\eta_{c}^{B}(L)$ in order to extract the critical packing fraction $\eta_{c}^{B}$ due to the corrections to scaling and stronger finite size effects.

\begin{tabular}{|c|c|c|c|c|}
\hline$\Delta$ & $\eta_{c}^{a v}$ & $\eta_{c}^{\max }$ & $\eta_{c}^{B}$ & $\eta_{c}^{\chi}$ \\
\hline 0.5 & $0.8141(5)$ & $0.8138(11)$ & $0.811(2)$ & $0.811(4)$ \\
\hline 1.0 & $0.8988(8)$ & $0.8976(20)$ & $0.896(4)$ & $0.895(5)$ \\
\hline 2.0 & $1.0215(18)$ & $1.0179(45)$ & $1.017(9)$ & $1.017(6)$ \\
\hline 4.0 & $1.147(4)$ & $1.141(5)$ & $1.141(5)$ & $1.140(5)$ \\
\hline$\infty$ & $1.228(3)$ & $1.222(6)$ & $1.224(8)$ & $1.225(5)$ \\
\hline
\end{tabular}

TABLE III: Numerical results for the critical packing fraction for the two dimensional NAHC mixtures for different nonadditivities and for the WR model $(\Delta=\infty)$. The numbers in parenthesis correspond to the error on the last digits.

ues obtained for finite sizes differ (see Fig. (4). However, the thermodynamic limit results for the critical packing fractions are consistent for all the four definitions. The estimation of the relative error which is smaller than $1 \%$ for all the critical packing fractions combines the error on the finite size estimates and the error coming from the finite size scaling to extract the thermodynamic limit results.

Let us now compare our results to previous simulations. Our prediction for the critical density $\rho_{c} \sigma_{A B}^{2}=$ $1.560(10)$ of the WR model is in good agreement with the value $1.566(3)$ obtained by Johnson et al. 3]. For the NAHC mixtures in two dimensions, the determination of the critical packing fractions have been recently obtained from numerical simulations by Saija and Giaquinta [10]. Only small non-addtivities $\Delta \leq 1$ have been considered and the system was limited to 800 particles. The critical packing fraction was determined without finite size scaling and may thus be considered only as a lower bound.
Their critical packing fraction obtained for $\Delta=1$ is $\eta_{c}=0.118(1+\Delta)^{2}=0.472$. A scaled particle theory predicts $\eta_{c}=0.096(1+\Delta)^{2}=0.385$ [27] whereas a virial expansion 0.324. The different definitions of the packing fraction in both cases explains the $\Delta$ dependence introduced to compare to our prediction $\eta_{c}=0.897(2)$. The strong difference should be contrasted by the fact that the prediction of a first-order perturbation theory usually referred to as MIX1 gives higher values for the critical packing fractions especially for large non-additivities $(\Delta \geq 0.4)[10]$. It could be interesting to pursue the comparison of this theory for higher non-additivities $(\Delta>1)$ with our numerical predictions.

\section{Three dimensional NAHC mixtures}

In three dimensions, we considered a large number of different non-additivities $\Delta=0.5,0.6,0.8,1.0,2.0,3.0$, 4.0 and 9.0 as well as the WR model $(\Delta=\infty)$. The system sizes ranged from $L=N_{A}^{1 / 3}=5$ to 50 . The largest systems contained $N_{A}+N_{B}=250000$ particles. Systems with up to $2 \times 10^{6}$ (or $L=100$ ) where simulated for some non-additivities. The equilibrium time was then close to the day on simple personal computers. Since a large number of densities needs to be simulated to extract the critical density, we are for $L=100$ at the limit of the numerical capabilities. As for the two dimensional systems, five consecutive runs were simulated. The first one was used to equilibrate the initial configuration and the four remaining ones served for the collection of data and the estimation of errors. $10^{5}$ to $10^{6}$ Monte-Carlo steps for each run were sufficient for the equilibration and in order to obtain a small error.

The modified susceptibility $\chi$ is determined as function of the packing fraction $\eta$ for different system sizes. It presents a single maximum $\chi_{\max }\left(\eta_{c}^{\chi}(L), L\right)$ at the finite size critical packing fraction $\eta_{c}^{\chi}(L)$. As can be seen on Fig. 5 this maximum depends on the system size with a power law behavior. From a linear fit of the maximum of the modified susceptibility as function of the system size in a log-log scale, we deduce the ratio of critical exponents $\gamma / \nu$ (see Table III). Those ratios for all non-additivities are systematically larger than the Ising universality class prediction but still inside the error bars. The relative errors were estimated as the sum of the average relative error on the maximum $\chi_{\max }$ and the error due to the linear fit.

The existence of corrections to scaling may explain the over-estimation of $\gamma / \nu$. Removing the data from the small size systems for the linear fits reduces the discrepancy with the Ising universality class prediction. A possible source for the corrections to scaling observed is the existence of fluctuations on the density inside each box during the simulations. Even though those fluctuations are small they may affect slightly the scaling behavior especially for small systems were the fluctuations are stronger. Some constrained simulations have been 


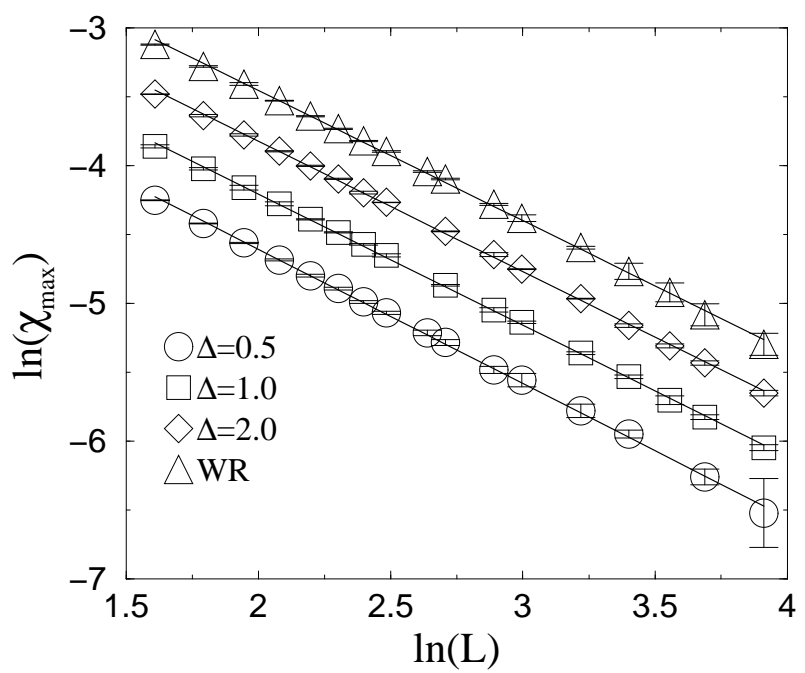

FIG. 5: Maximum of the modified susceptibility $\chi_{\max }$ as function of the system size $L$ in a log-log scale for different non-additivities $\Delta$ and for the WR model (Widom). The ratio of critical exponents $\gamma / \nu$ is extracted from linear fits. Data have been shifted for clarity.

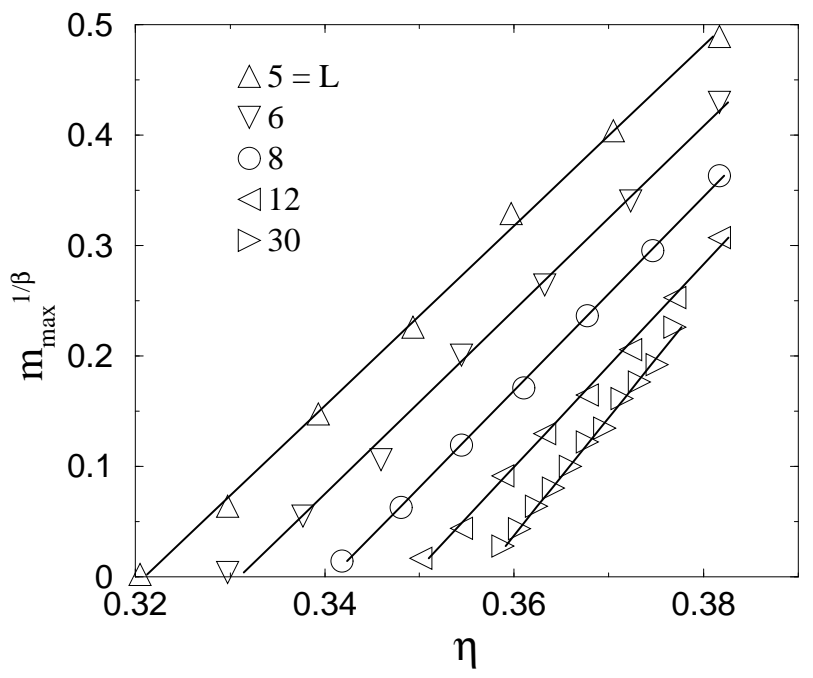

FIG. 6: Rescaled maximum of the distribution of the order parameter $m_{\max }^{1 / \beta}$ as function of the packing fraction $\eta$. The non-additivity considered is $\Delta=0.8$. The critical exponent $\beta=0.326$ predicted for the Ising university class has been used and the critical packing fractions $\eta_{c}^{\max }(L)$ for different system sizes $L$ are extracted from linear fits.

done were the density in each box was kept constant. The clusters were moved only if they did not change the total number of particles inside each box. This modification of the algorithm leads to similar results with less pronounced corrections to scaling. However, errors were also stronger since due to the rejections the equilibration time was sensibly increased.

The confirmation of the critical exponent $\beta$ is obtained
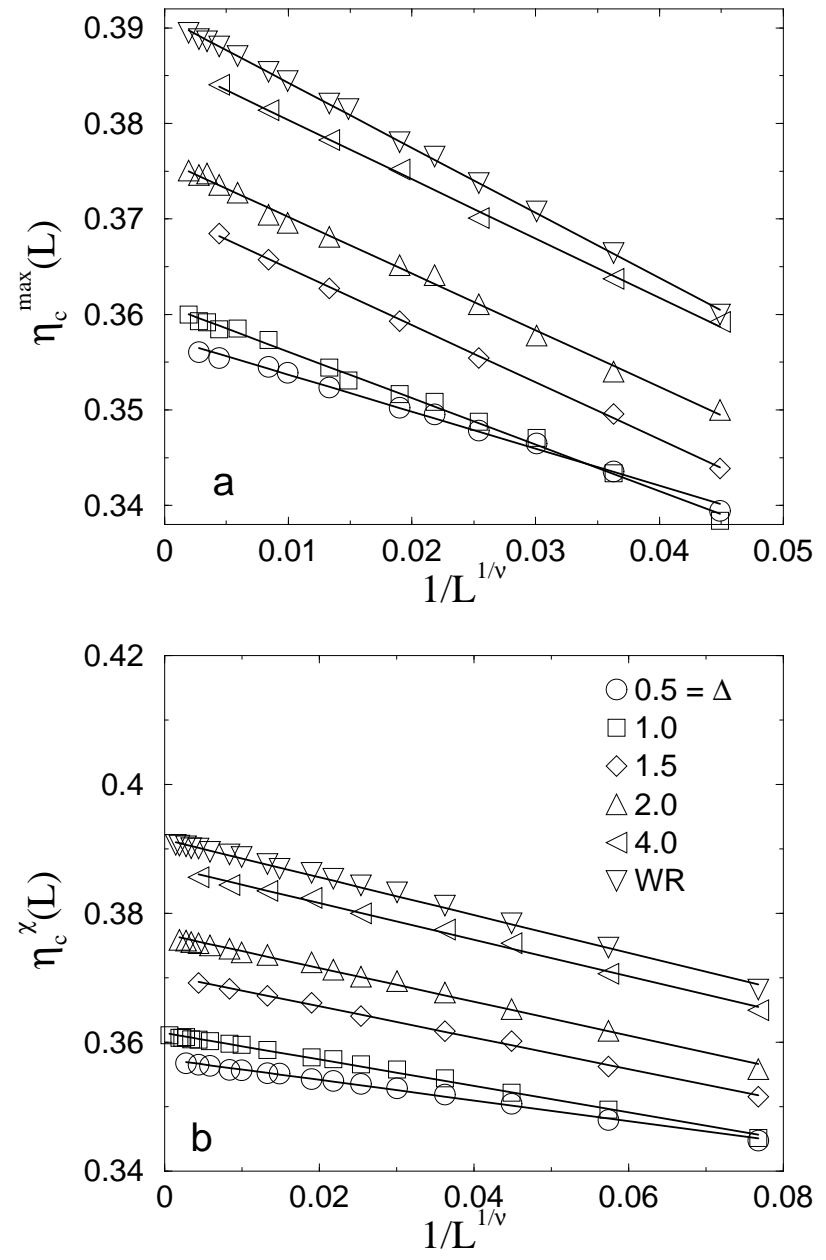

FIG. 7: Finite size critical packing fractions $\eta_{c}^{\max }(L)$ (a) and $\eta_{c}^{\chi}(L)(\mathrm{b})$ as function of the rescaled system size $1 / L^{1 / \nu}$ with $\nu=0.627$ as predicted by the Ising universality class. Different non-additivities are considered as well as the WR model (same legends for both figures). Linear fits of $\eta_{c}^{\max }$ as well as $\eta_{c}^{\chi}$ confirm the critical exponent $\nu=0.627$ and allow us to extract the critical packing fractions $\eta_{c}^{\max }$ and $\eta_{c}^{\chi}$.

from the plot of the rescaled maximum of the distribution of the order parameter $m_{\max }^{1 / \beta}$ as function of the packing fraction $\eta$ for different system sizes. A non-additivity $\Delta=0.8$ has been considered on Fig. [6 but similar results are obtained for all non-additivities and for the WR model. The linear behavior observed on the figure for a critical exponent $\beta=0.326$ confirms the Ising universality class prediction. The range of the linear regime is still rather small and limited for large packing fractions to $m_{\max }^{1 / \beta}<0.5$. The range of the linear regime for $\langle|m|\rangle^{1 / \beta}$ is even smaller and cannot allow us the confirmation of the critical exponent $\beta$ neither the determination of the finite size critical packing fraction $\eta_{c}^{a v}(L)$.

The critical packing fraction $\eta_{c}$ is determined from the plot of the finite size critical packing fraction $\eta_{c}(L)$ as function of the rescaled system size $1 / L^{1 / \nu}$ with the ex- 


\begin{tabular}{|c|c|c|c|}
\hline$\Delta$ & $\eta_{c}^{\max }$ & $\eta_{c}^{B}$ & $\eta_{c}^{\chi}$ \\
\hline 0.5 & $0.3577(3)$ & $0.3574(3)$ & $0.3574(3)$ \\
\hline 0.6 & $0.3572(4)$ & $0.3564(4)$ & $0.3568(4)$ \\
\hline 0.8 & $0.3583(5)$ & $0.3581(4)$ & $0.3580(5)$ \\
\hline 1.0 & $0.3610(5)$ & $0.3614(3)$ & $0.3614(5)$ \\
\hline 1.5 & $0.3706(5)$ & $0.3703(5)$ & $0.3705(8)$ \\
\hline 2.0 & $0.3758(5)$ & $0.3766(5)$ & $0.3766(8)$ \\
\hline 3.0 & $0.3827(10)$ & $0.3839(6)$ & $0.3843(9)$ \\
\hline 4.0 & $0.3862(10)$ & $0.3871(8)$ & $0.3872(11)$ \\
\hline 9.0 & $0.3896(9)$ & $0.3908(6)$ & $0.3914(9)$ \\
\hline$\infty$ & $0.3910(4)$ & $0.3912(4)$ & $0.3912(4)$ \\
\hline
\end{tabular}

TABLE IV: Numerical results for the critical packing fractions in three dimensions for the different non-additivities considered and for the WR model $(\Delta=\infty)$. The numbers in parenthesis correspond to the error on the last digits.

pected exponent $\nu=0.627$. The numerical results for $\eta_{c}^{\max }(L)$ (Fig. 7 $\mathrm{f}$ ) and for $\eta_{c}^{\chi}(L)$ (Fig. 7b) are plotted for different non-additivities and for the WR model. The error bars are smaller than the symbols and thus not represented. As can be seen, $\eta_{c}^{\max }$ and $\eta_{c}^{\chi}$ present a linear behavior with respect to $1 / L^{1 / \nu}$ for $\nu=0.627$. This confirms the value of the critical exponent $\nu$ predicted by the Ising universality class. The results are similar for $\eta_{c}^{B}$ and for the other non-additivities considered. The corrections to scaling observed for $\eta_{c}^{\chi}$ and $\eta_{c}^{B}$ in two dimensions are not present in the three dimensional systems. It is possible to notice a change in the slope of the linear fit around $\Delta=1$. Already evident on Fig. [7] this is confirmed by the results for the non-additivities $\Delta=0.6$ and 0.8 (data not shown on the figure). For $\Delta>1$ the slope is independent of the non-additivity whereas for $\Delta<1$ the slope is increasing with the nonadditivity. This change may be due to the onset of the fluid-solid transition that the model experience for large packing fractions. At sufficiently low non-additivity, the phase separation transition is expected to be pre-empted by the fluid-solid transition 27.

All the critical packing fractions for the different nonadditivities and for the WR model are presented on Table IV] The relative errors are less than $0.5 \%$ and the three definitions of the critical packing fraction $\eta_{c}^{\max }, \eta_{c}^{\chi}$ and $\eta_{c}^{B}$ lead to identical estimations in the thermodynamic limit.

Let us now compare our results with others. Our prediction for the critical density of the WR model is $\rho_{c} \sigma_{A B}^{3}=0.7470(8)$ which is in strong agreement with the value $0.748(2)$ obtained by Johnson et al. [3] with another cluster algorithm. Our result improves by a factor two the error on this critical density. The critical packing fraction has been determined from different numerical simulations for a large set of non-additivities. For a nonadditivity $\Delta=1$, our prediction $\eta_{c} /(1+\Delta)^{3}=0.04515(8)$ is slightly higher than the value $0.04484(20)$ proposed by Góźdź [12]. However, it strongly confirms the previ-

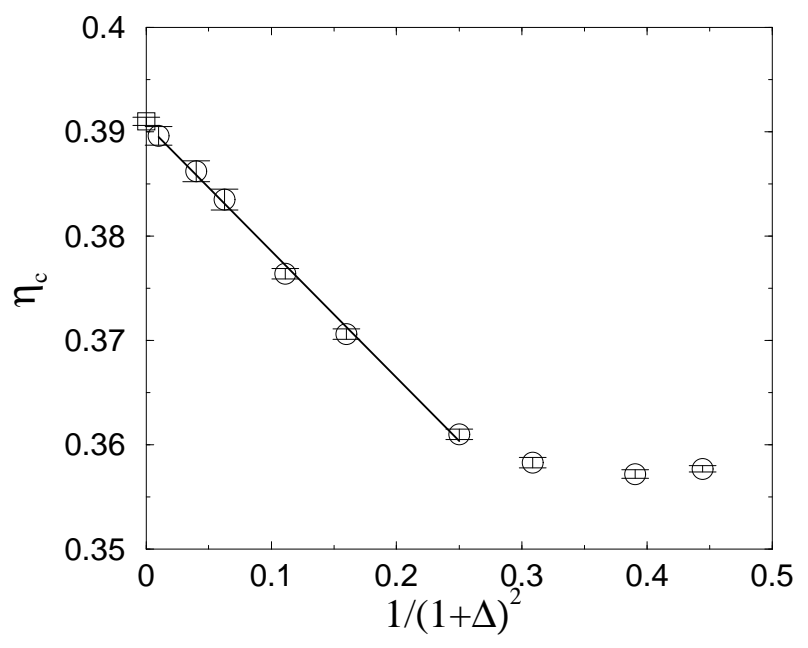

FIG. 8: Critical packing fraction for the different nonaddtivities $\eta_{c}(\Delta)$ as function of $1 /(1+\Delta)^{2}$ (disks) and for the WR model (square). The line corresponds to a linear fit for the six largest non-additivities and leads to an estimation of $\eta_{c}=0.3907(5)$ for the WR model.

ous under-estimation of 0.0288 by Saija et al. [9]. The non-additivity dependence introduced is due to a different definition for the packing fraction. Góźdź 12 also predicted the critical packing fraction $0.0866(2)$ and $0.0611(2)$ for the non-additivity $\Delta=0.6$ and 0.8 respectively. Its predictions compare nicely with our results $\eta_{c} /(1+\Delta)^{3}=0.0871(1)$ and $0.0614(1)$. It is important to notice that the critical packing fraction is determined to our knowledge for non-additivities $\Delta>1$ for the first time in this study. This is possible thanks to the absence of a critical slowing down of the simulations for large nonadditivities with the cluster algorithm in comparison to the simulations in the semigrand canonical ensemble.

From the determination of the critical packing fraction for large non-additivities, it is possible to consider the convergence of the NAHC mixtures to the WR model which corresponds to an infinite non-additivity. It is evident from Fig. 8 that the critical packing fraction $\eta_{c}(\Delta)=\eta_{c}(\infty)-B /(1+\Delta)^{2}$ for large non-additivities. A linear fit for the six largest non-additivities leads to an estimate of $\eta_{c}=0.3907(5)$ for the WR model in good agreement with the direct simulations. The coefficient $B$ was estimated to be 1.21. This interesting dependence of $\eta_{c}(\Delta)$ on $\Delta$ may be a good test for the different theories developed to determine the critical packing fraction of the NAHC mixtures.

The Binder parameter offers another test of the universality class from its intersection value $U^{*}$. In Fig. 9] we have plotted the Binder parameter as function of the packing fraction for different system sizes and for the non-additivity $\Delta=2$. It can be seen that the intersections between the different curves occur at smaller and smaller values as the system size increases but still at higher values than the expected universal one $U^{*}=0.47$. 


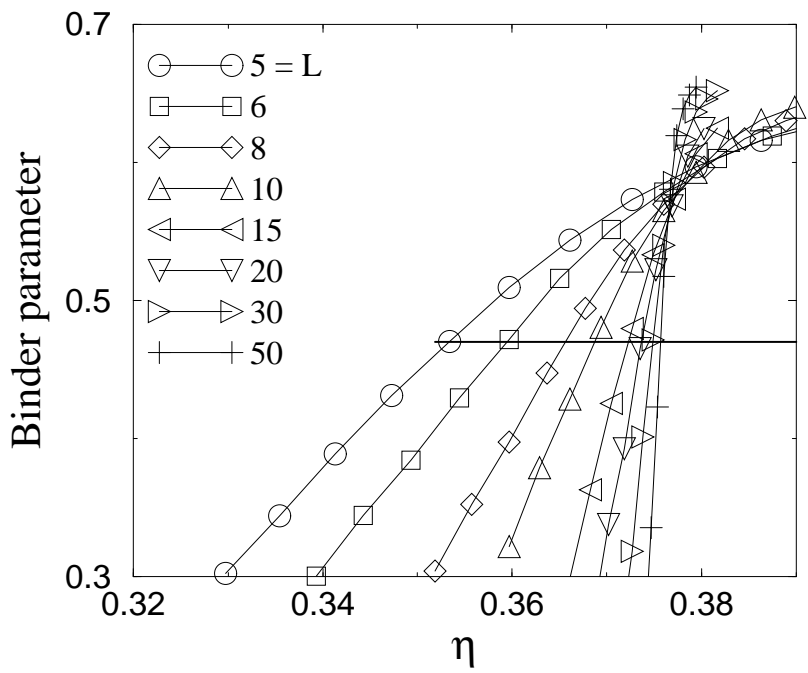

FIG. 9: Binder parameter for different system sizes as function of the packing fraction $\eta$ for the non-additivity $\Delta=2.0$. The horizontal line represents the universal intersection value $U^{*}=0.47$ expected for the Ising universality class.

Similar results are observed for the other non-additivities and for the WR model. This result was already observed in Góźdź simulations [12]. Corrections to scaling may explain such behavior. For our definition of the critical packing fraction $\eta_{c}^{B}$ based on the Binder parameter, the arbitrary choice was $U\left(\eta_{c}^{B}(L), L\right)=1 / 2>U^{*}$. Thus, $\eta_{c}^{B}(L)$ was expected to decrease with increasing system size. The opposite behavior is found. The absence of corrections to scaling in the dependence of $\eta_{c}^{B}(L)$ with the system size is thus an argument against this explanation for the high intersection in Fig. 9 However, no other explanations have been found.

\section{DISCUSSION}

In this paper we present a cluster algorithm for the numerical simulations of the NAHC mixtures as well as for the WR model. This cluster algorithm allows one to simulate large systems (up to $10^{6}$ particles). Each Monte-Carlo step corresponds to the non-local move of a large number of particles reducing strongly the equilibration time. The absence of critical slowing down for increasing non-additivities $\Delta$ allows us to study large nonadditivities and the convergence of the NAHC mixtures to the WR model when $\Delta \rightarrow \infty$.

Two and three dimensional systems have been considered. In both cases, the models were found to belong to the Ising universality class for all the non-additivities considered as well as for the WR model. The critical packing fractions $\eta_{c}$ have been determined from four different finite size definitions. All the definitions lead to identical predictions in the thermodynamical limit with a high precision.

Different theories have been used to determine the critical packing fractions $\eta_{c}(\Delta)$ of NAHC mixtures [26], from the scaled particle theory [27] to the virial expansion [28, 29] or the density functional theory [30]. A comparison with our precise numerical predictions of the critical packing fractions for a large set of non-additivities $\Delta$ is possible. Even if a quantitative comparison between theory and simulations is difficult, the $\Delta$ behaviour of the critical packing fraction $\left(\eta_{c}=\eta_{c}(\infty)-B /(1+\Delta)^{2}\right.$ in three dimensions) for large non-additivities $\Delta$ is a good test for a theory.

Recently, Jagannathan and Yethiraj [5] have studied the dynamical behavior of the WR model close to the phase separation transition. The cluster algorithm could be used to consider larger systems in order to be closer to the critical density. The cluster algorithm could also be used to study the stability and interfacial properties in confined geometries 31].

\section{Acknowledgments}

W. Krauth is thanked for useful discussions and a careful reading of the manuscript.
[1] B. Widom and J. S. Rowlinson, J. Chem. Phys. 52, 1670 (1970).

[2] C.-Y. Shew and A. Yethiraj, J. Chem. Phys. 104, 7665 (1996).

[3] G. Johnson, H. Gould, J. Machta, and L. K. Chayes, Phys. Rev. Lett. 79, 2612 (1997).

[4] P. C. Hohenberg and B. I. Halperin, Rev. Mod. Phys. 49, 435 (1977).

[5] K. Jagannathan and A. Yethiraj, Phys. Rev. Lett. 93, 015701 (2004).

[6] D. Gazzillo, G. Pastore and S. Enzo, J. Phys.: Condens. Matter 1, 3469 (1989).

[7] D. Gazzillo, G. Pastore and R. Frattini, J. Phys.: Condens. Matter 2, 8463 (1990).
[8] D. Frenkel, Physica A 263, 26 (1999).

[9] F. Saija, G. Pastore and P. V. Giaquinta, J. Phys. Chem. B 102, 10368 (1998).

[10] F. Saija and P. V. Giaquinta, J. Chem. Phys. 117, 5780 (2002).

[11] K. Jagannathan and A. Yethiraj, J. Chem. Phys. 118, 7907 (2003).

[12] W. T. Góźdź, J. Chem. Phys. 119, 3309 (2003).

[13] T. Biben and J.-P. Hansen, Phys. Rev. Lett. 66, 2215 (1991).

[14] A. Buhot and W. Krauth, Phys. Rev. Lett. 80, 3787 (1998).

[15] M. Dijkstra, R. van Roij and R. Evans, Phys. Rev. Lett. 82, 117 (1999). 
[16] D. A. Kofke and E. D. Glandt, Mol. Phys. 64, 1105 (1988).

[17] E. de Miguel, E. Martin del Rio and M. M. Telo da Gamma, J. Chem. Phys. 103, 6188 (1995).

[18] C. Dress and W. Krauth, J. Phys. A: Math. Gen. 28, L597 (1995).

[19] A. Buhot and W. Krauth, Phys. Rev. E 59, 2939 (1999).

[20] A. Buhot, Phys. Rev. Lett. 82, 960 (1999).

[21] L. Santen and W. Krauth, Nature 405, 550-551 (2000).

[22] R. H. Swendsen and J.-S. Wang, Phys. Rev. Lett. 58, 86 (1987).

[23] U. Wolff, Phys. Rev. Lett. 62, 361 (1989).

[24] J.-G. Malherbe and S. Amokrane, Mol. Phys. 97, 677 (1999).

[25] D. P. Landau and K. Binder, A Guide to Monte Carlo
Simulations in Statistical Physics, (Cambridge University Press, Cambridge, 2000).

[26] A. Santos, M. López de Haro and S. B. Yuste, cond-mat/0409430 (2004).

[27] R. M. Mazo and R. J. Beaman, J. Chem. Phys. 93, 6694 (1990).

[28] F. Saija and P. V. Giaquinta, J. Phys.: Condens. Matter 8, 8137 (1996).

[29] F. Saija, G. Fiumara and P. V. Giaquinta, J. Chem. Phys. 108, 9098 (1998).

[30] M. Schmidt, Phys. Rev. E 63, 010101(R) (2001); J. Phys.: Condens. Matter 16, L351 (2004).

[31] Y. Duda, E. Vakarin and J. Alejandre, J. Colloid Interface Sci. 258, 10 (2003). 